\title{
Mycobacterium haemophilum sp. nov., a New Pathogen of Humans $\dagger$
}

\author{
DAVID SOMPOLINSKY, ${ }^{1,2}$ ANNIE LAGZIEL, ${ }^{1}$ DAVID NAVEH, ${ }^{3}$ AND TULI YANKILEVITZ ${ }^{3}$ \\ Department of Microbiology, Asaf Harofe Government Hospital, Zerifin ${ }^{1}$; Rapaport Laboratories, Bar-Ilan \\ University, Ramat-Gan"; and Department of Internal Medicine "A," Meir Hospital, Kfar Saba, Israel
}

\begin{abstract}
A patient under immunosuppressive treatment of Hodgkin's disease developed generalized skin granulomata and subcutaneous abscesses. Several aspirated pus samples yielded acid-fast rods with the following properties: temperature optimum, about $30^{\circ} \mathrm{C}$ with no growth at $37^{\circ} \mathrm{C}$; slow growth ( 2 to 4 weeks); nonchromogenic; hemoglobin or hemin requirement for growth; catalase negative; pyrazinamidase and nicotinamidase positive; and urease negative. The guanine-pluscytosine content of the deoxyribonucleic acid was calculated from the melting temperature to be $66.0 \mathrm{~mol} \%$. It is concluded that these isolates belong to a new species, for which the name Mycobacterium haemophilum is proposed. The type strain of this species is strain 1 (= ATCC 29548). The new species is related to $M$. marinum and $M$. ulcerans.
\end{abstract}

Granulomatous skin diseases of humans caused by mycobacteria other than Mycobacterium tuberculosis and $M$. leprae are well known. The two organisms most often involved etiologically are $M$. marinum and $M$. ulcerans. Both species characteristically do not invade the viscera, have a low optimal temperature for growth $\left(30\right.$ to $\left.33^{\circ} \mathrm{C}\right)$, and do not develop at $37^{\circ} \mathrm{C}$ in primary culture. They grow on nutrient media (Loewenstein-Jensen, Petragnani, Middlebrook) in use for $M$. tuberculosis, although $M$. ulcerans may require an unusually long incubation period-up to 10 weeks-for primary isolation from clinical material. The colonies of $M$. ulcerans are eugonic, as are most virulent strains of $M$. tuberculosis; $M$. marinum grows rapidly (4 to 8 days) and produces smooth, cream-colored colonies that turn yellow when exposed to light. Infections with $\boldsymbol{M}$. marinum are generally acquired in swimming pools, hot springs, and rivers and by the handling of fish tanks. Skin ulcers caused by $M$. ulcerans occur exclusively in tropical zones, and the distribution of the organism in nature is unknown.

In this communication, a new acid-fast organism from skin granulomata is described. It has temperature limits for growth similar to those of $M$. ulcerans and $M$. marinum, but its nutritional requirements, growth characteristics, and biochemical activities differ from those described for any other known pathogenic mycobacterium.

$\uparrow$ Submitted as a tribute to the memory of Werner B. Schaefer, from the Division of Research, National Jewish Hospital, Denver, Colo.

\section{CASE HISTORY}

After World War II, a 27-year-old woman was diagnosed as having tuberculosis. She received treatment until 1951. In February 1969, at the age of 51, she was referred to the Department of Internal Medicine "A," Meir Hospital, Kfar Saba, Israel, because of malaise, anemia, fever, and weight loss. Several enlarged cervical lymph nodes were noticed, and biopsy showed diffuse reticulohistiocytic Hodgkin's disease. Lymphography indicated involvement of abdominal lymph nodes also. This ambulatory patient was treated with X-irradiation, steroids, and isoniazid.

In August 1971, the patient appeared again at the Department of Internal Medicine " $A$ " with complaints of painful swellings over the left elbow and knee and with a slightly elevated temperature. She was not aware of any mechanical trauma at the sites of the skin lesions. Thorough questioning did not produce any information of interest: she had lived in her flat in Nathanya uninterruptedly, had not bathed at the seashore or in any swimming pool, had had no contact with any fishes or aquaria, and had not worked in the garden or even grown plants in a pot; her house was infested with rats, but she was not aware of any direct contact with these or any other animals.

At admission, blood analysis revealed general pancytopenia, and the bone marrow was hypoplastic. A few hard nodules were present in the skin at her left elbow and knee. Histology of biopsy specimens showed minute necrotic foci in the lower dermis, surrounded by areas of infiltration with granulocytes, lymphocytes, monocytes, fusiform cells, and a few giant cells of the Langerhans type (Fig. 1). Bacteria, i.e., acidfast rods occurring singly or in small groups, some of them occurring clearly intracellularly in monocytes and giant cells, were seen only upon Ziehl-Neelsen staining.

The patient was released from the hospital since the efflorescences seemed to subside, but 2 months 67 


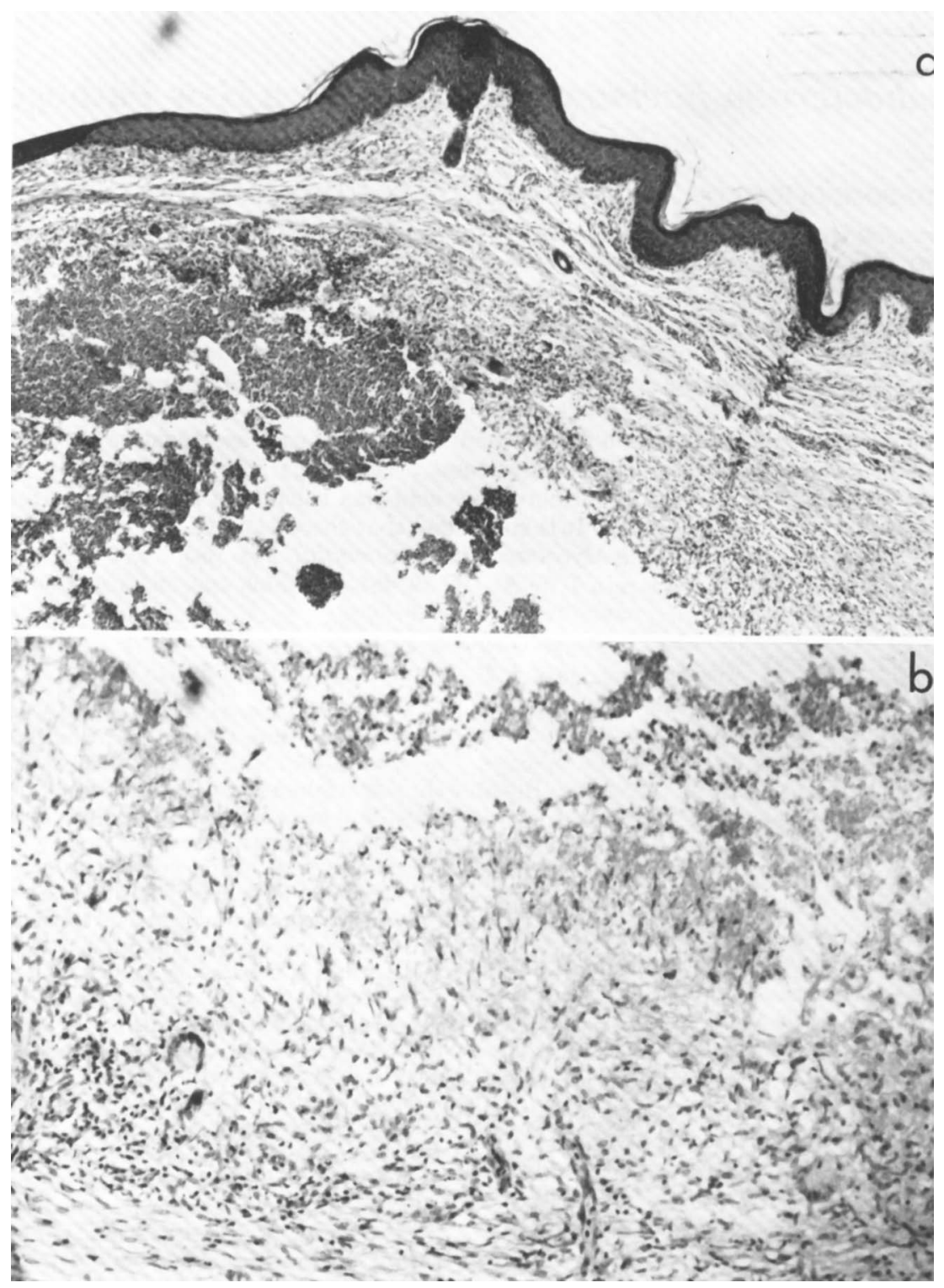

FiG. 1. (a) Necrotic granuloma of the skin. Hematoxylin and eosin. $\times 40$. (b) High magnification of granuloma wall. Note pallisading fibroblasts and multinuclear Langhans-type giant cell. Hematoxylin and eosin. $\times 250$.

that were larger and more painful than previously, some of them reaching the size of a dove's egg and fluctuating on palpation (Fig. 2). A viscous, yellowish to greenish pus, which on smear showed numerous granulocytes, lymphocytes, monocytes, and a few giant cells, was obtained by aspiration. Again, only acid-fast rods similar to tubercle bacilli were demonstrated.

The skin disease climaxed during October to December 1971. At one time, the patient was covered with more than 30 lesions of different size and development, from superficial skin infiltrations to fluctuat- ing abscesses and open fistulas, draining greenish purulent material. The majority of the lesions occurred on the extremities, but a few of them were found on the abdominal wall and the gluteal regions, and one was observed deep in the breast region. Regional lymph nodes seemed not to be involved, and the occurrence of the lesions did not indicate spread by lymphatic vessels.

Antimycobacterial therapy was initiated in October 1971: streptomycin, $1.0 \mathrm{~g}$ three times a week; ethambutol, 1,600 $\mathrm{mg}$ daily; and isoniazid, $300 \mathrm{mg}$ daily. The 


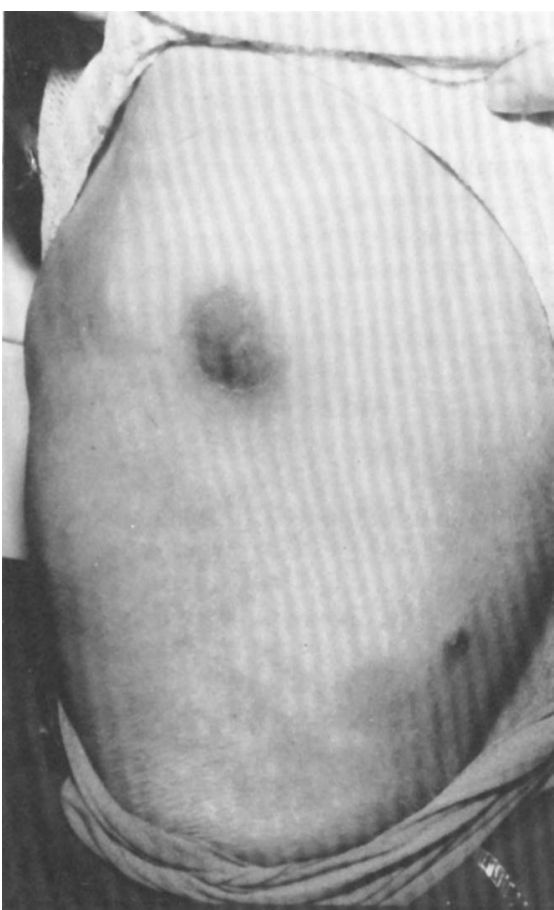

Fig. 2. Skin lesions of the patient. Shown are lesions in the initial state (areas of hyperemia), one well-developed abscess before fistulation, and one scar after fistulation.

abscesses were aspirated, and a $30 \%$ p-aminosalicylic acid ointment was applied superficially. Slowly, one after one, the skin lesions healed, some of them leaving scars, and new efflorescences occurred less frequently. Finally, about 6 months after the initiation of antituberculosis treatment, the patient was entirely free from skin lesions.

During the next year, the patient was admitted to the hospital a number of times for respiratory tract infections, each time without signs of recurrence of her skin disease. During the last hospitalization period, her blood analysis showed an extreme pancytopenia, and eventually she died with signs of generalized sepsis. Necropsy was not performed.

\section{MATERIALS AND METHODS}

Bacterial strains. Four separate isolates of the new organism, strains 1 through 4, were studied. For comparison, the following strains of mycobacteria were also studied: $M$. ulcerans NCTC 10417, $M$. marinum NCTC 10011, and $M$. chelonei subsp. abscessus NCTC 946, which were obtained from the National Collection of Type Cultures, The Public Health Laboratory Service Board, London, England; $M$. avium D (avian tuberculin production strain, Central Veterinary Laboratory, Weybridge, England), which was donated by Aliza Kohn, The Veterinary Institute, Beth Dagan; and two strains of $\boldsymbol{M}$. intracellulare (no. 8 and 9), which were isolated by $R$. Levith, Smuel Harofe Hospital, Beer Yacov, Israel.
Culture media. Dubos oleic acid agar, cornmeal glycerol agar, brain heart infusion agar, and Middlebrook 7H10 agar were purchased from Difco Laboratories, Detroit, Mich. The 7H10 agar medium was usually enriched with OADC (per $100 \mathrm{ml}$ of medium oleic acid, $5 \mathrm{mg}$; bovine albumin, fraction $\mathrm{V}, 0.5 \mathrm{~g}$; glucose, $0.2 \mathrm{~g}$; catalase, $0.4 \mathrm{mg}$; and $\mathrm{NaCl}, 0.085 \mathrm{~g}$ ) from Difco Laboratories, Detroit, Mich. This medium was poured into petri dishes, which were sealed with tape during the period of incubation. Other media used included Sula defined medium, Loewenstein-Jensen medium, Sabouraud glucose agar, blood agar, and chocolate agar; they were prepared as described by Cruickshank et al. (2). Requirement of hemoglobin and hemin for growth was checked on LoewensteinJensen medium, Middlebrook $7 \mathrm{H} 10$ agar, and 7H9 liquid medium enriched with OADC. To a part of these media was added $0.4 \%$ hemoglobin or 2 to 60 $\mu \mathrm{M}$ hemin, and the cultures were incubated at 25,30 , and $37^{\circ} \mathrm{C}$. The following compounds were examined for stimulation of growth in $7 \mathrm{H9}$ medium at $30^{\circ} \mathrm{C}$ : hematoporphyrin, sodium pyruvate, sodium sulfite, sodium thioglycolate, $\mathrm{FeCl}_{3}$, and activated charcoal. Colonies on Middlebrook 7H10 agar with $60 \mu \mathrm{M}$ hemin and Loewenstein-Jensen medium with $0.4 \%$ hemoglobin were exposed to fluorescent and incandescent light, at a distance of $15 \mathrm{~cm}$ from the light source, for examination of photochromogenicity.

Morphology. To determine the dimensions of the bacteria, a Zeiss screw-micrometer eye-piece was used. The bacteria were from 3-week-old colonies on 7H10 agar with $60 \mu \mathrm{M}$ hemin. Both unstained bacteria (phase-contrast microscopy) and Ziehl-Neelsenstained organisms were measured.

Biochemical activities. Catalase production, niacin synthesis, nitrate and tellurite reductions, hydrolysis of Tween 80 , production of arylsulfatase, iron uptake, and tolerance to $\mathrm{NaCl}$ were studied as described by Runyon et al. (11); the neutral red test was performed as suggested by Hughes et al. (6); the reagents for the examinations for urease, pyrazinamidase, and nicotinamidase were prepared by the method of Georges and Dailloux (5); these tests, as well as the examination for beta-galactosidase, were performed as described by Wayne et al. (12). The production of phosphatase was determined according to hydrolysis of phenolphthalein phosphate. A $1 \%$ concentration of this reagent was incorporated into Middlebrook $7 \mathrm{H} 9$ broth enriched with ADC (Difco) and $60 \mu \mathrm{M}$ hemin. The broth was inoculated with a turbid suspension of the test organism, and phosphatase activity was tested for after 3 days of incubation by the addition of 1 drop of $25 \% \mathrm{NH}_{4} \mathrm{OH}$. For the detection of indole production, growth on Middlebrook 7H10 agar slants containing $60 \mu \mathrm{M}$ hemin and $1 \%$ tryptophane was examined. The cultures were incubated with paper strips impregnated with Ehrlich reagent, and cultures of Escherichia coli were used as positive controls.

Base composition of DNA. The guanine-plus-cytosine $(G+C)$ content of deoxyribonucleic acid (DNA) was calculated from the thermal melting point of the DNA. The bacteria were crushed in a French press, and the DNA was prepared essentially as described by Baess (1). After incubation with ribonuclease and 
extraction of the enzyme protein by two chloroformisoamyl alcohol treatments, DNA was precipitated with $95 \%$ ethanol; the isopropyl alcohol was omitted. The DNA was eventually dissolved in a 0.1 dilution of the saline-sodium citrate buffer $(0.1 \times \mathrm{SSC})$ and was essentially free from protein, as shown by light adsorption at $280 \mathrm{~nm}$ versus that at $260 \mathrm{~nm}$. The thermal melting point was determined in a Gilford adsorption spectrophotometer, model $2400-\mathrm{S}$, with a thermocouple inserted into the blank cuvette $(0.1 \times \mathrm{SSC})$ and with simultaneous recording of temperature and optical density for the blank and the three test samples. The cuvette holder was heated with circulating hot water from a thermostat-controlled water bath, with automatic regulation of a temperature increment of 1 Celsius degree every $4 \mathrm{~min}$.

Enzymes and chemicals. Catalase, hemoglobin, and pancreatic ribonuclease $A$ were of bovine origin, and hemin of equine origin, and were purchased from Sigma Chemical Co., St. Louis, Mo. Most other chemicals used, including hematoporphyrin, sodium citrate, sodium pyruvate, sodium thioglycolate, and activated charcoal, were from the same source.

\section{RESULTS}

The new isolates were short, occasionally slightly curved, rods that occurred singly or in minor cordlike formations. They were strongly acid fast and alcohol fast as demonstrated by the Ziehl-Neelsen procedure. They were not stainable by the Gram method. By microscopic inspection of bacterial suspensions, no active motility was observed. The dimensions of the rods were determined with a 3-week-old culture of strain 1 on $7 \mathrm{H} 10$ agar enriched with $60 \mu \mathrm{M}$ hemin. By phase-contrast microscopy of live cells, the mean size was 0.6 by $2.4 \mu \mathrm{m}$, with the range 0.4 to 0.7 by 1.4 to $3.2 \mu \mathrm{m}$. For bacteria that were fixed and stained by the Ziehl-Neelsen method, the dimensions 0.4 by $1.6 \mu \mathrm{m}$ were obtained, with the size ranging from 0.3 to 0.5 by 1.2 to $2.2 \mu \mathrm{m}$. Occasionally a longer, threadlike organism was observed among the relatively short rods.

Further characteristics of the new isolates are presented in Tables 1 and 2.

The following description may be of help in the future for isolating and identifying this organism.

Primary isolation, colonial morphology, and nutritional requirements. During the 4 months after the appearance of the granulomata in the patient, many unsuccessful attempts to culture the acid-fast rods seen on smears were made both in the local hospital laboratory and in a specialized Tuberculosis Department of a Public Health Laboratory. A considerable number of pus samples were inoculated on diverse media, including Loewenstein-Jensen, Dubos oleic acid agar, Sula defined fluid medium, Sabouraud dextrose agar, and cornmeal glycerol agar. The inoculated media were kept at 30,37 , and $43^{\circ} \mathrm{C}$ under aerobic and anaerobic conditions, but no visible growth occurred in 3 months. Likewise, intraperitoneal injection of pus samples into guinea pigs failed to produce pathological changes.

Later, in a renewed attempt to isolate the organism, pus samples were again inoculated on a number of nutrient media, including those specific for mycobacteria, brain heart infusion agar, blood agar (7\% washed sheep erythrocytes), and McLeod chocolate agar. The cultures were incubated at room temperature and at 32 and $37^{\circ} \mathrm{C}$. Six to eight weeks later, growth of numerous, grayish-white, smooth and rough colonies occurred on all of the chocolate agar slants incubated at $32^{\circ} \mathrm{C}$ but not on any of the other media. On three later occasions, pus samples inoculated in a similar way yielded growth only on chocolate agar at $32^{\circ} \mathrm{C}$.

Cultures maintained for 3 years by serial transfer behaved similarly to those obtained on primary isolation. Growth was obtained on a variety of nutrient media provided that hemolyzed sheep erythrocytes were added to the medium; the addition of whole erythrocytes did not stimulate growth. Hemolyzed blood could be substituted by $0.4 \%$ hemoglobin or $60 \mu \mathrm{M}$ hemin (Fig. 3 and 4). With $7 \mu \mathrm{M}$ hemin, colonies were pin-point in size; with $2 \mu \mathrm{M}$, no growth was obtained. The following could not replace hemin for growth stimulation in Middlebrook 7H9 medium: hematoporphyrin (7.5 to $60 \mu \mathrm{M})$, sodium sulfite $\left(0.8\right.$ to $1.6 \mathrm{mM}$ ), $\mathrm{FeCl}_{3}$ ( 7.5 to 60 $\mu \mathrm{M})$, sodium thioglycolate $(0.8$ to $1.6 \mathrm{mM})$, sodium pyruvate ( 3 to $30 \mathrm{mM})$, and activated charcoal $(0.05$ to $0.20 \mathrm{mg} / \mathrm{ml})$. Likewise, growth was not obtained on Middlebrook $7 \mathrm{H} 10$ agar, even when enriched with OADC containing active catalase, unless hemoglobin or hemin was added; neither did the addition of $4 \mu \mathrm{g}$ of catalase (bovine liver) per $\mathrm{ml}$ stimulate growth without the addition of hemin.

Colonies of the new isolate on Middlebrook 7H10 agar enriched with OADC plus hemin are drier than the smeary growth of $M$. marinum but not as crumbly as those of $M$. ulcerans. Within these limits, there are differences corresponding to smooth-rough variations between colonies even on the same plate. The colonies are nonpigmented and remain so even when exposed to a strong light source that induces pigmentation of colonies of $M$. marinum.

Serial subculture over a period of 3 years likewise did not alter the temperature limits for the growth of this new isolate. The organism developed colonies on Middlebrook $7 \mathrm{H} 10$ agar plus OADC plus $60 \mu \mathrm{M}$ hemin during 2 to 4 weeks of incubation at $32^{\circ} \mathrm{C}$; under similar con- 

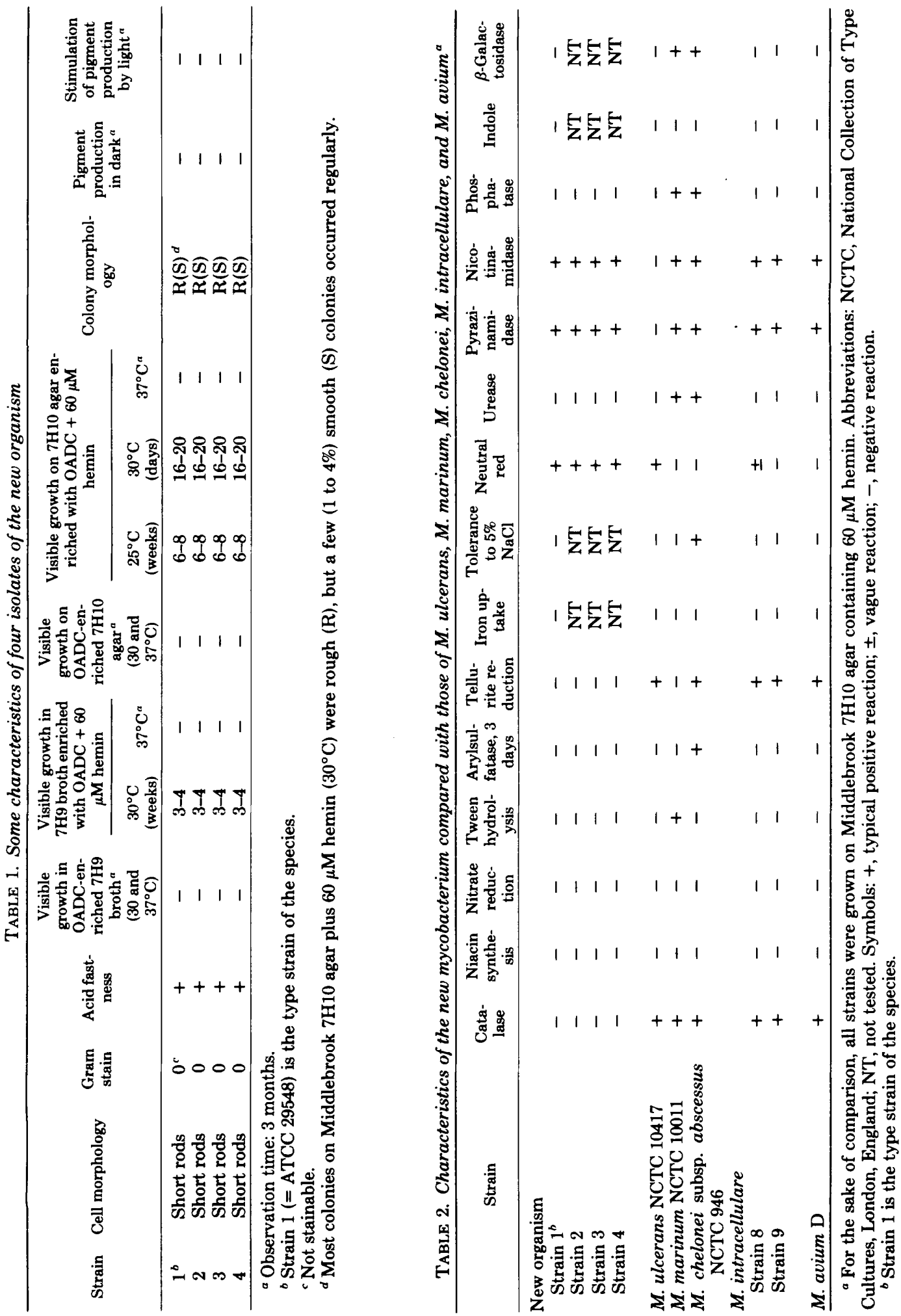


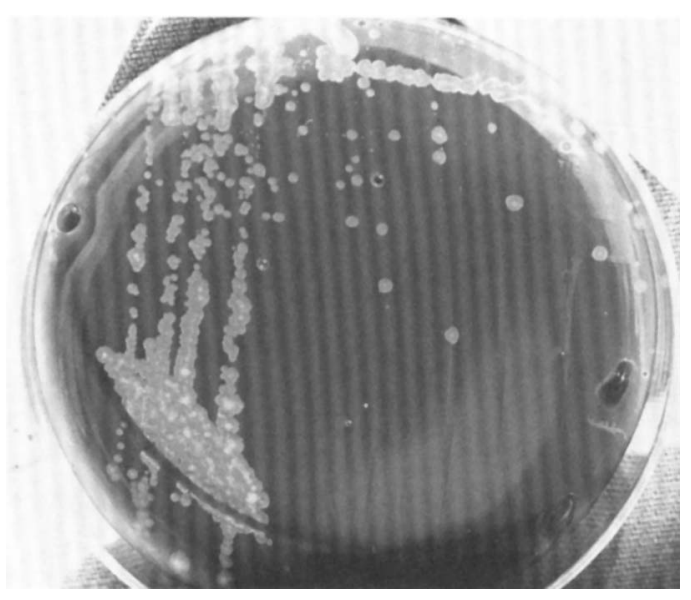

FIG. 3. Colonies of the new organism on Middlebrook agar with $60 \mu \mathrm{M}$ hemin after 4 weeks of incubation at $32^{\circ} \mathrm{C}$.

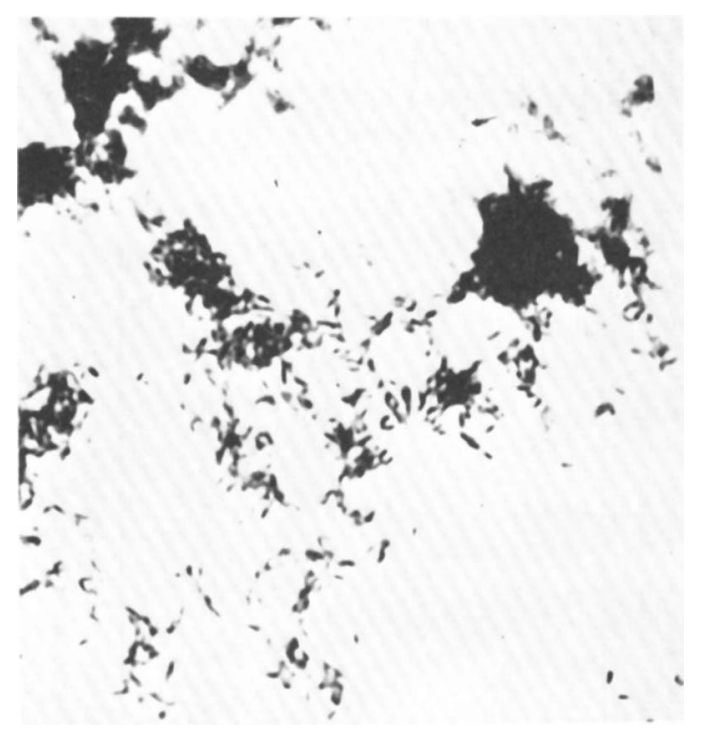

FIG, 4. Ziehl-Neelsen-stained preparation of cells from one of the colonies depicted in Fig. 3. $\times 1,200$.

ditions, $\boldsymbol{M}$. marinum and $\boldsymbol{M}$. ulcerans develop colonies in 8 to 10 days and 8 to 10 weeks, respectively. At 25 and $35^{\circ} \mathrm{C}$, the incubation time is doubled, and at $37^{\circ} \mathrm{C}$ no growth occurs during 3 months of incubation.

Biochemical activities. The isolates were catalase negative (Table 2). The neutral red test was positive for the new isolate and for $M$. ulcerans but negative for the other organisms examined. The new organism is also similar to $M$. ulcerans in being $\beta$-galactosidase negative, urease negative, and phosphatase negative. It is similar to $\boldsymbol{M}$. marinum in hydrolyzing pyrazin- amide and nicotinamide and in failing to reduce tellurite. It can, therefore, be concluded that, in its biochemical activities, the organism differs essentially from both $M$. marinum and $M$. ulcerans. Most of the enzymatic activities shown in Table 2 gave identical results for the new organism and the strains of $M$. intracellulare and $M$. avium examined, but, in contrast to the $M$. avium-M. intracellulare complex, it did not produce catalase or tellurite reductase. $M$. chelonei subsp. abscessus NCTC 946 differs from the new organism in catalase, urease, arylsulfatase, tellurite reductase, and $\beta$-galactosidase productions.

Base composition of DNA. The members of the genus Mycobacterium can be divided into two groups on the basis of the $\mathrm{G}+\mathrm{C}$ contents of their DNAs: one has a $\mathrm{G}+\mathrm{C}$ content of 63 to 66 $\mathrm{mol} \%$, and the other has a content of 67 to 70 mol\% (13). $M$. marinum belongs to the first of these groups, and the $M$. avium-M. intracellulare complex belongs to the other. Since the growth temperature of the new organism is similar to that of $M$. marinum, whereas a great number of its enzymes correspond to those of $M$. avium and $M$. intracellulare, a determination of the $\mathrm{G}+\mathrm{C}$ ratio of its DNA seemed of particular interest. The base composition was calculated from the thermal melting point $\left(T_{m}\right)$ of the DNA. The curve of the thermal denaturation of the DNA of the new isolate was similar to that of $M$. marinum NCTC 10011 but different from that of $M$. intracellulare strain 8 (Fig. 5). After correction for thermal expansion, the $\mathrm{G}+\mathrm{C}$ content was determined according to the following formula: $\mathrm{G}+\mathrm{C} \mathrm{mol} \%=\left(T_{m}-53.9\right) \times$ 2.44 (1). The $\mathrm{G}+\mathrm{C}$ contents were calculated as $65.0 \mathrm{~mol} \%$ for $M$. marinum NCTC $10011,66.0$ $\mathrm{mol} \%$ for the new organism, and $69.8 \mathrm{~mol} \%$ for $M$. intracellulare strain 8.

Susceptibility to chemotherapeutic agents. Table 3 summarizes the susceptibility of the new isolate to various drugs. This organism is highly resistant to isoniazid, streptomy$c i n$, and ethambutol but is susceptible to $p$-aminosalicylic acid. The organism is inhibited by $100 \mu \mathrm{g}$ of $p$-nitrobenzoic acid per $\mathrm{ml}$ in spite of the fact that niacin is not synthesized (Table 2); this combination of characters is unusual (4).

Animal inoculation. Inoculations of heavy suspensions of the organism injected intravenously, intramuscularly, and subcutaneously into mice and guinea pigs did not cause obvious pathological changes, and most of the animals survived the 3-month observation period. Some of the mice died, however, within 2 to 4 weeks after inoculation, and large numbers of the mycobacteria could be found in smears of liver, kidneys, and spleen, most of them intracellularly 


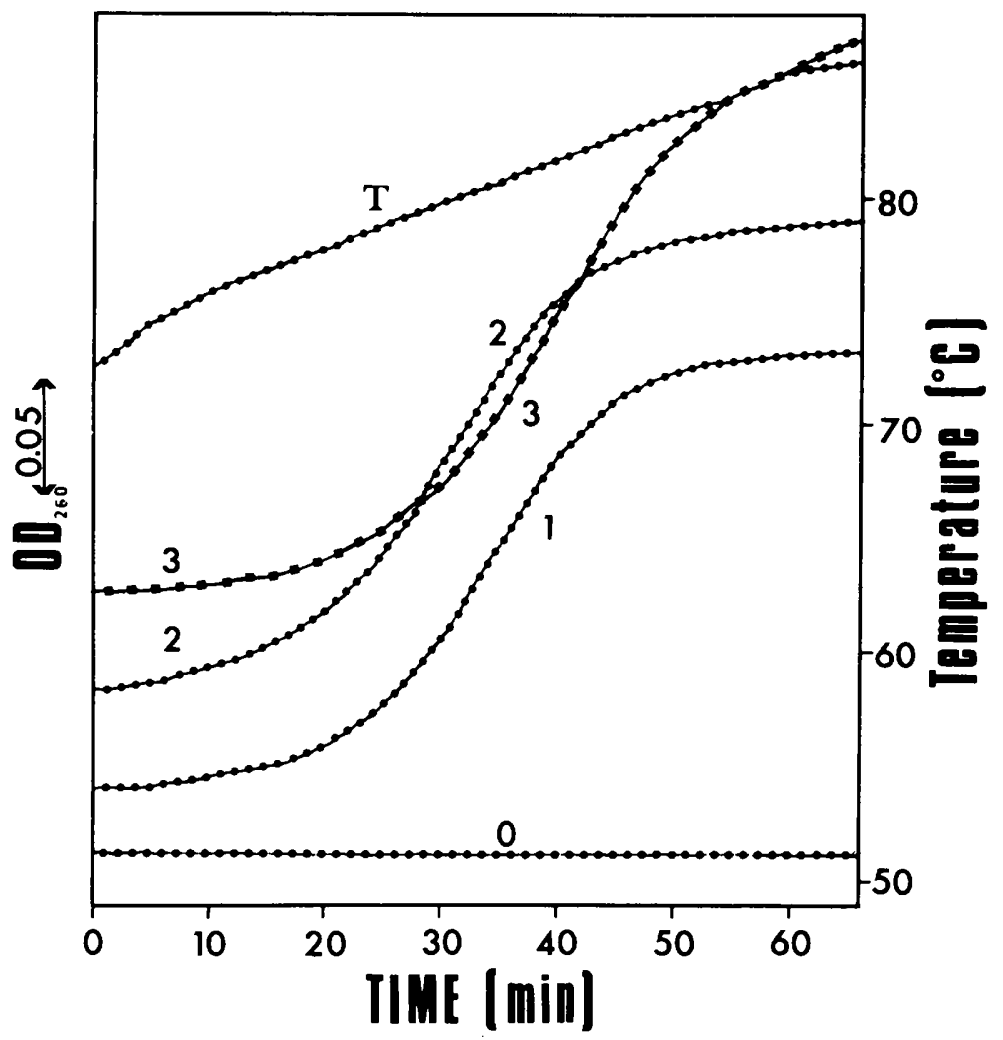

FIG. 5. Thermal denaturation of DNA from: (1) the new organism, (2) M. marinum NCTC 10011, and (3) $M$. intracellulare strain 8. All DNA samples were of an initial optical density at $260 \mathrm{~nm}\left(O \mathrm{D}_{260}\right)\left(25^{\circ} \mathrm{C}\right)$ of 0.6. " 0 " indicates the blank cuvette. T, Temperature.

TABLE 3. Susceptibility of strain 1 of the new organism to antimicrobial agents

\begin{tabular}{|c|c|c|}
\hline \multirow[t]{2}{*}{ Antimicrobial agent ${ }^{a}$} & \multicolumn{2}{|c|}{$\begin{array}{c}\text { Concn of drug }(\mu \mathrm{g} / \mathrm{ml}) \\
\text { at which the new orga- } \\
\text { nism is: }\end{array}$} \\
\hline & Resistant & Susceptible \\
\hline Isonicotinic acid hydrazide & 25 & 100 \\
\hline Streptomycin sulfate & 10 & 50 \\
\hline$p$-Aminosalicylic acid & 0.5 & 2 \\
\hline Ethambutol hydrochloride & 125 & 500 \\
\hline Kanamycin sulfate & 5 & 25 \\
\hline Capreomycin sulfate & 5 & 25 \\
\hline$p$-Nitrobenzoic acid & 20 & 100 \\
\hline
\end{tabular}

${ }^{a}$ The drugs were incorporated into Middlebrook 7H10 agar supplemented with OADC enrichment (Difco Laboratories, Detroit, Mich.) and $60 \mu \mathrm{M}$ hemin (equine type III crystalline, Sigma Chemical Co., St. Louis, Mo.).

in monocytes or macrophages; macroscopically, however, these organs were unchanged.

When rabbits were given heavy intravenous suspensions of heat-inactivated mycobacteria, the tissue surrounding the injection site of $\boldsymbol{M}$. ulcerans developed severe granulomatosis that caused obliteration of the blood vessels after a few injections. Histology showed the acid-fast rods, singly and in groups, to be present outside and inside monocytes and macrophages. A similar, but far less pronounced, reaction was seen in rabbits injected with suspensions of the new mycobacterium but not in those injected with $M$. marinum NCTC 10011. Intramuscular injection of $10^{6}$ to $10^{7}$ cells of the new organism into the thigh of frogs (Bufo sp. and Rana sp.) was without effect when the animals were kept at room temperature, but, when kept at $30^{\circ} \mathrm{C}$, the animals died within 8 to 20 days, and clumps of bacteria were found in smears of liver and kidneys.

\section{DISCUSSION}

The following is a summary description of the new organism. They are straight rods that stain uniformly acid fast by the Ziehl-Neelsen procedure. They are not stainable by the Gram method. Nonpigmented colonies on Middlebrook $7 \mathrm{H} 10$ agar develop within 2 to 4 weeks at $30^{\circ} \mathrm{C}$ and are predominantly rough, with smooth variants occurring frequently. The organism 
grows slowly at $25^{\circ} \mathrm{C}$ and does not grow at $37^{\circ} \mathrm{C}$. It is an obligate aerobe. The organism requires hemoglobin or hemin for growth on artificial media. It is catalase and urease negative and does not reduce nitrate and tellurite; however, it is positive for pyrazinamidase and nicotinamidase. The organism is resistant to isonicotinic acid hydrazide. Four strains were isolated from subcutaneous abscesses of a woman under treatment for Hodgkin's disease. Its habitat is unknown.

The most outstanding feature of $\boldsymbol{M}$. ulcerans and $M$. marinum is their temperature relationship. No other known human pathogens have an optimal growth temperature so low and are unable to grow at normal body temperature. The organism described in this paper is clearly a third member of this notable group of mycobacteria. Although the new organism has more enzymatic reactions in common with those of the $M$. avium-M. intracellulare complex than those of $M$. marinum and $M$. ulcerans, it is felt that a psycrophile should not be classified with species that grow at $40^{\circ} \mathrm{C}$ and often even at $45^{\circ} \mathrm{C}$. Growth temperature is one of the most stable characters of bacterial species, and Marks (9) has recently proposed a "new practical" classification system of mycobacteria with growth temperature as the basic fundamentum divisionis.

The $\mathrm{G}+\mathrm{C}$ ratio of the DNA supports our contention that the new organism should not be grouped together with $M$. avium-M. intracellulare strains (13). M. marinum has a relatively low $\mathrm{G}+\mathrm{C}$ content, similar to that of the new organism; so far we have not examined $M$. $u l$ cerans for its $\mathrm{G}+\mathrm{C}$ content, nor have we found in the literature a $\mathrm{G}+\mathrm{C}$ value for this organism. From a taxonomic point of view, pathogenesis may be a subordinate, but not an entirely useless, character. Whereas the results of inoculations of the new organism into mice may recall the Yersin-type reaction that $M$. avium produces, the natural disease caused by this organism resembles neither tuberculosis nor infections with $\boldsymbol{M}$. intracellulare. The disease does, however, have significant features in common with skin lesions caused by $M$. marinum and $M$. ulcerans. For $M$. marinum, the preference for a relatively low temperature could be related to a natural aquatic habitat. On the other hand, the natural reservoir of $\boldsymbol{M}$. ulcerans has not been demonstrated, and, in the case of the new organism, there is no clue to its origin.

However, the nutritional requirement of the new organism for hemoglobin or hemin argues against a purely saprophytic natural habitat. This requirement recalls certain isoniazid-resistant mutants of $M$. tuberculosis described two decades ago $(3,7,10)$. These mutants and the new organism described here are characterized by resistance to isoniazid, lack of catalase, and nutritional requirement for hemin. In the case of the new organism, isolation was from a patient under treatment with isoniazid. For the mutants of $M$. tuberculosis, hemin could be replaced by catalase or activated charcoal (7), but this was not the case with our isolate.

From a taxonomic point of view, the temperature relationships of this new organism serve to place it as a third member of a complex containing $M$. ulcerans and $M$. marinum. The enzymatic activities of the new organism (together with still unpublished results of serological examinations) amply confirm that the organism does not belong to either $M$. ulcerans or $M$. marinum. Therefore, we regard this organism as belonging to a new species, for which, because of its nutritional requirement for hemoglobin or hemin, we propose the name $M y$. cobacterium haemophilum (hae.mo'phi.lum Gr. n. haema blood; Gr. adj. philos loving; M.L. adj. haemophilus blood-loving). The type strain of $M$. haemophilum is strain 1 ; a culture of this strain has been deposited in the American Type Culture Collection (ATCC) under the number 29548.

It should be mentioned that a case of skin ulcers with acid-fast bacteria that resisted attempts at isolation was described a few years ago, but the authors did not indicate the media used in the isolation attempts (8). We have also been informed (G. Wellish, personal communication) about a case similar to ours in another Israeli hospital, but no attempts were made to isolate the organism on a medium with hemolyzed blood or hemin. Hopefully, the present communication will stimulate the use of a variety of nutritional supplements and incubation temperatures in similar situations in the future.

\section{ACKNOWLEDGMENTS}

We thank Aryeh Rozenszajn and Moshe Mayan for kind cooperation and help. We also wish to express our gratitude to M. Edelman of The Weizmann Institute of Science for advice on preparing the DNA for $T_{m}$ determination, and Dina Heller for help with the spectrophotometer.

This study was sustained by a Research Grant of the Ministry of Health. D.S. also received economic assistance as an Established Investigator of the Chief Scientist's Bureau of the Ministry of Health, Israel.

\section{REPRINT REQUESTS}

Address reprint requests to: David Sompolinsky, E. Rapaport Professor of Medical Microbiology, Bar-Ilan University, Ramat-Gan, Israel.

\section{LITERATURE CITED}

1. Baess, I. 1974. Isolation and purification of deoxyribonucleic acid from mycobacteria. Acta Pathol. Microbiol. Scand. Sect. B 82:780-784. 
2. Cruickshank, R., J. P. Duguid, B. P. Marmion, and R. H. A. Swain. 1975. Medical microbiology, vol. 2, 12th ed. Churchill Livingstone, Edinburgh.

3. Fisher, M. W. 1954. Hemin as a growth factor for certain isoniazid-resistant strains of Mycobacterium tuberculosis. Am. Rev. Tuberc. 69:797-805.

4. Gangadharam, P. R., and A. J. Droubi. 1973. Susceptibility of mycobacteria to $p$-nitrobenzoic acid in relation to their niacin production. Am. Rev. Respir. Dis. 108:143-146.

5. Georges, J. C., and M. Dailloux. 1973. Activités amidasiques quantitatives des mycobacteries atypiques. Ann. Biol. Clin. 31:217-224.

6. Hughes, D. E., E. S. Moss, M. Hood, and M. Henson. 1954. Virulence of Mycobacterium tuberculosis. Am. J. Clin. Pathol. 24:621-625.

7. Knox, R. 1955. Hemin and isoniazid resistance of $M y c o$. bacterium tuberculosis. J. Gen. Microbiol. 12:191-202.

8. Lomvardias, S., and G. E. Madge. 1972. Chaetoconidium and atypical acid-fast bacilli in skin ulcer. Arch. Dermatol. 106:875-876.

9. Marks, J. 1976. A new practical classification of the mycobacteria. J. Med. Microbiol. 9:253-261.

10. Middlebrook, G. 1954. Isoniazid-resistance and catalase activity of tubercle bacilli. Am. Rev. Tuberc. 69:471-472.

11. Runyon, E. H., A. G. Karlson, G. P. Kubica, and L. G. Wayne. 1974. Mycobacterium, p. 148-174. In E. H. Lennette, E. H. Spaulding, and J. B. Truant (ed.), Manual of clinical microbiology, 2nd ed. American Society for Microbiology, Washington, D.C.

12. Wayne, L. G., H. C. Engbaek, H. W. B. Engel, S. Froman, W. Gross, J. Hawkins, W. Käppler, A. G. Karlson, H. H. Kleeberg, I. Krasnow, G. P. Kubica, C. McDurmont, E. E. Nel, S. R. Pattyn, K. H. Schröder, S. Showalter, I. Tarnok, M. Tsukamura, B. Vergmann, and E. Wolinsky. 1974. Highly reproducible techniques for use in systematic bacteriology in the genus Mycobacterium: tests for pigment, urease, resistance to sodium chloride, hydrolysis of Tween 80, and $\beta$-galactosidase. Int. J. Syst. Bacteriol. 24:412-419.

13. Wayne, I. G., and W. M. Gross. 1968. Base composition of deoxyribonucleic acid isolated from mycobacteria. $J$. Bacteriol. 96:1915-1919. 